\title{
IDENTIDADES ESQUEMÁTICAS DE GÉNERO EN LA ESCUELA: A PROPÓSITO DEL PRIMER ANIVERSARIO DE LA MUERTE DE PIERRE BOURDIEU
}

\section{Schematic identities of gender in the school: Regarding the first anniversary of the death of Pierre Bourdieu}

\section{L'identités schématiques du genre à l'école: À propos $d u$ premier anniversaire de la mort du Pierre Bourdieu}

- José Vicente PEÑA CALVO y M. ${ }^{a}$ del Carmen RODRÍGUEZ MENÉNDEZ Universidad de Oviedo. Facultad de Ciencias de la Educación. Departamento de Ciencias de la Educación. C/. Aniceto Sela, s/n. 33005 Oviedo.

Fecha de aceptación definitiva: marzo de 2003 BIBLID [(1130-3743) 14, 2002, 235-263]

RESUMEN

El artículo que los autores presentan, a propósito del primer aniversario de la muerte del sociólogo francés Pierre Bourdieu, realiza una exposición de algunas ideas conceptuales existentes en su teoría de la práctica social, en particular las implicaciones de su noción de habitus para explicar el proceso de constitución de la identidad de género. A partir de este marco teórico, se presentan algunas propuestas de interés que pueden servir para ayudar a elaborar el armazón conceptual de una teoría de la educación que, en sus análisis de la influencia de la escuela en la formación de la identidad personal, tenga en cuenta al género como elemento configurador de la misma.

Palabras clave: habitus de género, identidades de género esquemáticas, espacio escolar de socialización, coeducación, Teoría de la Educación. 


\section{SUMMARY}

The paper that the authors present, with regard to the first anniversary of the death of French sociologist Pierre Bourdieu, makes a exhibition of some existing conceptual ideas in its theory of the social practice, in individual the implications of its notion of habitus in order to explain the process of constitution of the identity of gender. From this theoretical frame, some proposals of interest appear that can serve to help to elaborate the conceptual framework of a theory of the education that, in his analyses of the influence of the school in the configuration of the personal identity, takes in to consideration the gender as a configuration element of the same one.

Key words: gender's babitus, schematic identities of gender, socialization scholar space, coeducation, Theory of Education.

\section{SOMMAIRE}

L'article qui les auteurs proposent à propose du premier anniversaire de la mort du sociologue française, Pierre Bourdieu, il présente une exposition des quelques idées conceptuelles qui existent à leur théorie de la pratique sociale, en particulier les implications de sa notion de habitus pour expliquer les procès de constitution de la identité de genre. On présente quelques idées intéressantes à partir de cadre théorique, qui peuvent être utiles pour élaborer l'assemblage conceptuelle d'une théorie d'éducation qui rend compte du genre comme l'élément configurateur de l'influence de l'école à la formation de la identité personnelle.

Mots clef: Habitus de genre, identités de genre schématiques, espace écolier de socialisation, coéducation, Théorie de l'Éducation.

\section{INTRODUCCIÓN}

Al cumplirse un año de la muerte del sociólogo francés Pierre Bourdieu resulta oportuno y pertinente rescatar algunos de los aspectos más importantes de su producción científica e investigadora, más concretamente algunas nociones básicas que fundamentan su teoría de la práctica social. En este sentido, hemos de señalar su amplia elaboración teórica, y es que «sus análisis van desde la cultura y la política hasta el sindicalismo y las huelgas pasando por la moda, la literatura, el lenguaje y la música" (Alvarez-Uría y Varela, 1986, 252). Pero sin lugar a dudas la gran aportación que realiza es que sus trabajos inauguran una nueva forma de hacer sociología, al tiempo que efectúa un esfuerzo por presentar una teoría compacta sobre la práctica social o ciencia del conocimiento práctico. Ello ha llevado a diversos autores (Álvarez Sousa, 1996; Wacquant, 1994; García Canclini, 1998) a afirmar que su pensamiento se ha convertido en una teoría sobre la sociedad en general. Pronunciándose en la misma línea destaca Van Haecht (1999), para quien Bourdieu realiza un proyecto ambicioso y complejo que se plasma en una teoría general de 
la cultura, que toma su sentido en una sociología de las clases sociales más sofisticada que la desarrollada por los sociólogos marxistas. En suma, "Bourdieu ha desarrollado el cuerpo de una teoría social merecedora de una discusión detallada por derecho propio" (Jenkins, 1993, 67. La traducción es nuestra).

En el último año hemos estudiado la influencia de la escuela, en cuanto ámbito de socialización secundario, en el desarrollo de identidades de género adaptadas a los estereotipos que, desde la sociedad, se establecen para cada uno de los géneros. En las páginas que siguen no mostramos los resultados de esta investigación, pues ello excedería los límites marcados para la realización del presente artículo. Lo que sí presentamos son algunas reflexiones teóricas que han sustentado el desarrollo de la investigación. Dichas reflexiones toman como punto de partida la teoría de la práctica social de Pierre Bourdieu con el objetivo de estudiar las relaciones de género que se construyen en el interior de las instituciones educativas.

Para el logro de este objetivo hemos seguido los siguientes pasos:

1. Comenzamos con una aproximación conceptual al entramado teórico que sustenta los análisis de Bourdieu sobre el modo en que las personas construimos el mundo que nos rodea y las interacciones que establecemos con las personas de nuestro entorno. Para ello tomamos como referencia su noción de babitus.

2. A continuación, aplicando el principio de transferencia analógica, usamos el concepto de habitus, proporcionándole una nueva dimensión; de manera que dicho término se convierte en el elemento clave para entender el proceso de constitución de la identidad de género.

3. Ya por último, utilizamos el marco teórico elaborado con el objetivo de obtener un conjunto de elementos susceptibles de ayudar a configurar una Teoría de la Educación. Teoría de la Educación que debe tener como objetivo fundamental comprender el proceso de formación a través del cual se construye la identidad personal (García Carrasco y García del Dujo, 2001). Desde esta perspectiva, establecemos unas consideraciones de carácter general de cara a elaborar una Teoría de la Educación que tenga en cuenta el género como elemento de la identidad personal que se van configurando en el interior del sistema escolar.

\section{Algunos elementos teóricos de interés en la obra de Pierre Bourdieu}

Bourdieu elabora un modelo explicativo de cómo la vida social es construida cotidianamente por las personas en el seno del espacio social. En la base de ese modelo se encuentra una visión estratégica de la práctica social, la cual supone comprender que las acciones emprendidas por las personas en su vida cotidiana están guiadas por una especie de sexto sentido, denominado por Bourdieu: sentido práctico. De forma que el sentido práctico se definiría como una aptitud para moverse, actuar y orientarse en el mundo social basada en un conjunto de saberes no formulados de forma explícita. Precisamente será ese carácter implícito de los 
saberes que orientan la acción el que le lleve a afirmar que éstos no acceden a la conciencia de la persona que se guía por ellos. En este sentido, afirma que nuestro sentido práctico orienta «unas elecciones que no son menos sistemáticas por no ser deliberadas y que, sin estar ordenadas y organizadas con relación a un fin, no dejan por ello de poseer una especie de finalidad retrospectiva" (Bourdieu, 1991, 113). Así, en nuestro conocimiento del mundo social a través de un sentido práctico, que actúa alejado de la propia conciencia, el tipo de estrategias que utilizamos para orientar nuestra acción no han de ser conscientes ni racionales (aunque sí pueden ser razonables), pues las personas sabemos "sin saber" lo que tenemos que hacer, en algún lugar en el fondo de nuestras mentes existe un patrón usual de cómo las cosas son y ocurren, pero éste no accede a la conciencia.

En clara continuidad con el concepto sentido práctico se configura la noción de habitus $^{1}$. A riesgo de simplificar en exceso los postulados bourdieunianos podríamos conceptualizar el habitus como ese conjunto de saberes implícitos que guían el sentido práctico. Usando sus propias palabras el babitus se define como el "sistema de disposiciones durables y transferibles —estructuras estructuradas predispuestas a funcionar como estructuras estructurantes- que integran todas las experiencias pasadas y funciona en cada momento como matriz estructurante de las percepciones, las apreciaciones y las acciones de los agentes ante una coyuntura o acontecimiento y que él contribuye a producir» (citado por Sánchez de Horcajo, 1979,88$)^{2}$. En suma, el habitus como conjunto de esquemas internalizados que constituyen la condición de toda acción, de toda elección individual y que fundamenta la aptitud para moverse en el mundo social, es decir, el sentido práctico.

A partir de ello afirma que el habitus está constituido por esquemas internalizados que se definen por ser categorías no pensadas, durables y transferibles. Durables porque los esquemas o disposiciones del habitus, aprendidos en los primeros años de vida, son casi inmunes al paso del tiempo; y decimos casi inmunes porque están continuamente afectados por las experiencias nuevas a las que se ve abocada una persona todos los días de su vida. Como destaca García Inda (1995), el habitus es duradero pero no inmutable ${ }^{3}$. Y transferibles, lo que le permite

1. Aunque la noción de habitus ha sido elaborada por Bourdieu antes que su idea de "sentido práctico", hemos preferido comenzar con el análisis de esta última pues opinábamos que el discurso resultante estaba dotado de una mayor claridad conceptual.

2. Cita textual que Sánchez de Horcajo extrae de la siguiente publicación: Bourdeu, P. (1972) Esquisse d'une théorie de la pratique, précéde de trois études d'etbnologie Kabyle. Génova, Droz.

3. Bourdieu intenta superar una de las dicotomías tradicionales en el ámbito disciplinar de la sociología, la dicotomía acción/estructura. Desde esta perspectiva, destaca que hay dos dimensiones inseparables de lo social: las estructuras objetivas y los fenómenos subjetivos. De modo que afirma que las dos a proximaciones, estructuralista y constructivista, son dos momentos complementarios del mismo movimiento. El resultado de todo ello es una praxeología social (WACQUANT, 1994) conocida con el nombre de "constructivismo estructuralista" o "estructuralismo constructivista". Ello supone que los sujetos realizamos construcciones particulares de lo que para nosotros significa la vida social; sin embargo, esta construcción está determinada por el lugar que ocupamos en el espacio social. Es decir, las personas 
argumentar que las disposiciones que conforman el babitus facultan a la persona para producir efectos parecidos en campos de la práctica muy diversos, tendiendo a conferir regularidad a sus acciones. Es decir, aunque a partir del babitus se generen prácticas y conductas variadas, todas ellas se guían por el mismo principio explicativo, lo que les confiere una homogeneidad implícita. Ahora bien, también es preciso destacar que el habitus tiene "parte ligada con lo impreciso y lo vago" (Bourdieu, 1996, 84), de lo que se deduce que las disposiciones del habitus también pueden generar acciones imprevistas ${ }^{4}$.

Por otra parte, comentar que aunque las disposiciones del babitus se encuentran tan arraigadas en nuestro cuerpo y en nuestra mente que se tiene la impresión de haber nacido con esas disposiciones; estas formas de pensar, obrar y sentir no son, ni mucho menos, innatas puesto que son el resultado de un proceso de socialización. En este sentido, Bourdieu destaca la importancia de la posición que la persona ocupa en el espacio social en la formación del habitus, ya que es a través de las experiencias formadoras de la primera infancia, de toda la historia colectiva de la familia y de la influencia del mundo social que la rodea el modo en que el habitus se va formando. Los principios de percepción y pensamiento son continuamente reforzados, por acciones y discursos producidos por las personas que rodean al individuo, de forma inconsciente en la mayoría de las ocasiones y conscientemente en unas pocas.

Con relación al último aspecto destacado, Bourdieu afirma que la inculcación del habitus se puede realizar de forma explícita e implícita. Puede ser un aprendizaje por simple familiarización o mimetismo, en donde los esquemas del habitus se adquieren de forma inconsciente; o también pueden formarse a través de una transmisión explícita y expresa, mediante prescripciones y preceptos. El aprendizaje familiar del habitus, que supone una interiorización inconsciente, es mucho más eficaz que el aprendizaje institucionalizado, el cual supone un mínimo de racionalización que le hace disminuir su eficacia. Lo que se aprende inconscientemente es más eficaz y duradero que aquello que supone una toma de conciencia sobre el propio proceso de apropiación. Por ello, la inculcación "familiar", que se realiza de forma implícita, genera que los esquemas de percepción y acción sean interiorizados de una manera casi natural, provocando que el habitus se convierta en una «especie de dominio adquirido que funciona con la seguridad automática de un instinto" (Bourdieu, 1991, 176). A partir de ello, Bourdieu establece que la

construimos la realidad, pero esa construcción está constreñida por coacciones estructurales, por lo que las disposiciones del habitus aunque son duraderas (influjo de la estructura) no son inmutables (importancia de la construcción personal).

4. Con frecuencia se ha criticado el carácter determinista y mecanicista de la noción de babitus, por cuanto su definición "excluye toda recreación de un espacio para la libertad y la responsabilidad" (VAN HAEChT, 199, 45). Dado que excede los límites marcados para el presente artículo presentamos, a continuación, una serie de referencias primordiales para analizar los postulados críticos: VAN HAECHT (1999), Evens (1999), Jenkins (1993), Martínez Sanmartí y Pérez Sola (1997), Bourdieu y Wacquant (1994). 
acción pedagógica primaria, realizada fundamentalmente en el seno de la familia, conformará un habitus primario, que se revelará como el principio de evaluación de toda experiencia posterior. A esta idea nosotros unimos la importancia de la escuela, en cuanto agente secundario de socialización, en la configuración de los esquemas del babitus.

3. Contribución de las tesis de P. Bourdieu para el estudio del proceso de ConstiTUCIÓN DE LA IDENTIDAD DE GÉNERO

A partir de las tesis teóricas que hemos descrito queremos introducir una nueva, y creemos novedosa, mirada al proceso de construcción de la identidad de género. Así, a partir de la noción de babitus, sugerimos, por analogía, un nuevo concepto: el habitus de género. Desde nuestro punto de vista, dicho concepto desarrolla un papel primordial en el proceso de constitución de la identidad de género porque nos permite comprender cómo se construye el género, al ser definido como el sistema de esquemas incorporados que da estructura a las conductas, actitudes y formas de sensibilidad que hacen que una persona se sienta y perciba como masculina o femenina. Hombres y mujeres están dotados de una matriz de disposiciones y competencias capaces de generar una amplia variedad de acciones que están en consonancia con lo que la sociedad establece como deseable y adecuado para cada uno de los géneros. De este modo, el babitus de género aparece como un principio que permite apreciar y percibir el mundo de una manera determinada y orientar las prácticas y conductas de una persona de acuerdo a su género de pertenencia.

A partir de aquí consideramos que existe un babitus masculino y un habitus femenino, en cuanto esquemas que guían las acciones de hombres y mujeres respectivamente en la dirección del estereotipo de género marcado por la sociedad. Ello nos permite concluir que esos sistemas de esquemas deben estar dotados de unas características definitorias que marcan la acción de la persona en la dirección establecida, configurando la base de la identidad de género. Veamos, a continuación, cuáles son esas propiedades que definen el babitus de cada género y que, como tales, están orientando el sentido de nuestras prácticas y acciones ${ }^{5}$.

Sin ninguna duda, en el núcleo del habitus de género masculino reside la idea de poder, mientras que la configuración del habitus femenino se fundamenta en la

5. Hemos de señalar que Bourdieu ha publicado durante la década de los 90 dos obras referidas a la dominación masculina (véase una reseña de las mismas en las referencias bibliográficas finales). En ambas publicaciones destaca la existencia de un babitus viril masculino y de un habitus sumiso femenino. Habitus que se define en función de la situación dominante ocupacla por los hombres en las relaciones que se establecen entre los géneros. Nosotros, sin embargo, hacemos un uso más amplio del término "babitus de género", pues no restringimos su significado al análisis de la dominación masculina, sino que es una noción básica para el proceso de constitución de la identidad de género. 
JOSÉ VICENTE PEÑA CALVO Y M. a DEL CARMEN RODRÍGUEZ MENÉNDEZ

ausencia de éste. Por tanto, la conformación del habitus masculino se asienta en la detección del poder y en su carácter de sujeto trascendente, en palabras de S. de Beauvoir; por el contrario, el babitus femenino se fundamenta en la carencia de poder y en su configuración como mero objeto.

Partiendo de este marco de referencia, ¿Qué propiedades definen la peculiaridad concreta del habitus masculino? En primer lugar, destacamos el carácter viril de este habitus, en el sentido de que el conjunto de disposiciones que lo conforman se definen por la exaltación de los valores masculinos y por su asociación con el valor físico o moral, lo que impone a cada hombre el deber de afirmar su virilidad. Si bien esta virilidad se definía en el pasado a partir de sus actividades cazadoras y guerreras, en la actualidad dicha afirmación pasa por la adquisición de un estatus preponderante en la esfera pública. Por tanto, un habitus viril como disposición a acrecentar los valores propios, que se relaciona directamente con lo que T. Moi (1991) ha denominado como una illusio masculina, entendiendo por tal expresión la creencia de que uno mismo es importante (self-importance). Para Bourdieu, este habitus viril es consecuencia de un proceso de socialización que inclina a los hombres a tomarse solemnemente los juegos que el mundo social constituye como serios y a jugarlos seriamente, tal sería el caso de la guerra, del trabajo desempeñado en la esfera pública e incluso del deporte. Para Bourdieu (1999 y 2000) esta virilidad actúa, además, como una carga por cuanto el hombre se siente obligado a estar siempre a la altura de las circunstancias, buscando la distinción en la esfera pública. Esta obligación, autoimpuesta puede ser la causa del fenómeno descrito por Gil Calvo (1997), para quien el género masculino atraviesa, en la actualidad, una etapa de crisis originada porque el arquetipo viril autoritario se encuentra en desuso; al tiempo que no ha sido sustituido por otros modelos de referencia. En esta situación los hombres se encuentran incompetentes y desautorizados, no saben adoptar nuevos papeles por lo que la obligación de estar a la altura no siempre se cumple.

A este babitus viril hay que unir la disposición a ejercer la dominación en las relaciones humanas; en suma, un babitus dominante. En este sentido, Bourdieu destaca la existencia de una libido dominandi, que se define como "deseo de dominar a los otros hombres y, secundariamente, a título de instrumento de lucha simbólica, a las mujeres" (Bourdieu, 1999, 28). Esta libido dominandi se inculca ya durante la primera infancia, pues se incita $o$, al menos, se tolera que los niños se muestren agresivos en sus juegos, estableciéndose altas dosis de competitividad entre ellos, al tiempo que también intentan dirigir y dominar los comportamientos de sus compañeras. De ahí que esta libido dominandi es lo que hace que los hombres "estén socialmente formados e instruidos para dejarse atrapar, como unos niños, en todos los juegos que les son socialmente atribuidos y cuya forma por excelencia es la guerra" (Bourdieu, 2000, 96).

6. En suma, se produce en los hombres una disposición (habitus) a incidir en las maneras de pensar y sentir de los otros, utilizando para ello diversos mecanismos de dominación que no han de ser

(c) Ediciones Universidad de Salamanca

Teor. educ. 14, 2002, pp. 235-263 
Una tercera propiedad que define el habitus de género masculino es su disposición a la imparcialidad y objetividad ${ }^{7}$, de manera que puede ser calificado como habitus impersonal. Ello supone, en primer lugar, que los hombres aprecian peligrosas aquellas situaciones que implican una afiliación personal íntima. Los hombres, y como afirma V. J. Seidler, han aprendido a hablar con la voz imparcial de la razón, de manera que la "voz de un hombre asume un tono de objetividad e imparcialidad convirtiéndose en una voz impersonalizada, una voz que tiene autoridad porque no pertenece a nadie en particular, mientras proclama, al mismo tiempo, que respeta a todos" (citado en Morgan, 1999, 107). Esto trae como consecuencia, y siguiendo a Gil Calvo, que la inhibición expresiva de las emociones en el género masculino es resultado de un pudor emocional que les lleva a proteger su intimidad. Además, otra característica del habitus impersonal es que los hombres se sienten totalmente seguros en momentos de logro individual, por lo que destacan una ética de derechos basada en la igualdad, que se centra en la comprensión de la imparcialidad. En suma, un conjunto de esquemas (babitus) impersonales e instrumentales que orientan a la adquisición y manifestación de dotes de mando, ambición y autocontrol. Como afirman Echebarría Echabe y Valencia Gárate (1993), estos rasgos instrumentales se relacionan con las transformaciones analizadas por Weber en el desarrollo del capitalismo incipiente. Dicho capitalismo trajo consigo el desarrollo de una concepción ideológico-religiosa que lo legitimara $^{8}$. Este marco ideológico estimulará una visión individualista de la persona que será percibida como autónoma, al tiempo que el éxito personal se atribuirá única y exclusivamente al logro personal. Así el agente socializado en esta ética será una persona con necesidad de logro personal, competitiva y ambiciosa. Los autores mencionados concluyen que dado que este sistema de valores es especialmente funcional en el ámbito público, son los hombres los que han sido socializados con mayor frecuencia para la consecución de estas actitudes.

necesariamente conscientes. Ahora bien, también destacamos que, en señalados momentos sociohistóricos, se han introducido variaciones en las disposiciones asociadas al habitus. En este sentido, queremos poner el acento en la excepción hecha por Bourdieu a la existencia universal del babitus dominante. Destaca, así, que el habitus judío tradicional en los países de la Europa Central a finales del siglo xIX supuso una inversión del habitus masculino como disposición dominante, por cuanto se trataba de una comunidad en la que el rechazo a la violencia, incluso en sus formas más ritualizadas como es el caso del deporte, desvalorizó la agresividad y favoreció el desarrollo de tendencias suaves. Ello pone de manifiesto que solamente un análisis detallado de las distintas épocas sociohistóricas puede arrojar luz sobre las variaciones, mayores o menores, que nos podemos encontrar en las disposiciones de los respectivos babitus de género.

7. Para la definición de esta tercera propiedad nos asentaremos en el corpus teórico desarrollado por Carol Gilligan. Para un análisis detallado de esta interesante teoría acudir a: Gilıgan, C. (1985) La moral y la teoría. Psicología del desarrollo femenino. México, Fondo de Cultura Económica (trabajo original publicado en 1982).

8. No en el sentido que la religión prescribe el capitalismo, sino en el carácter complementario y de mutuo apoyo que ambas formaciones presentan y en el alto grado de compatibilidad de algunos valores. 
Por lo que respecta al género femenino, la histórica falta de poder de las féminas, así como la posición subordinada que han ocupado en sus relaciones sociales ha marcado la configuración de su babitus. En este sentido, la primera propiedad que lo caracteriza es la sumisión. Por tanto, definimos un babitus sumiso que, siendo resultado de una experiencia precoz y prolongada en el seno de interacciones penetradas por estructuras de dominación, orienta su conducta. Esta disposición sumisa ha dirigido la percepción del mundo de las féminas, así como las conductas que han generado y su actitud ante el mundo que las rodea. En este sentido, la abnegación, la resignación, el silencio, la abstención, la abstinencia, la docilidad o la entrega han sido definidas como virtudes típicamente femeninas. Además, la disposición sumisa ha provocado que las mujeres demuestren más conflictos de culpabilidad relacionados con la agresividad de su propia conducta o de la de otras personas. Les es más difícil aceptarla y reconocerla y esta incapacidad se acompaña de sentimientos conflictivos y ansiedad. Tannen (1996) destaca que observando con detalle a niños y niñas mientras juegan, se puede concluir que los chicos pelean cuando quieren jugar, mientras que las chicas evitan las discusiones aun cuando están realmente en desacuerdo.

Otra de las consecuencias de este babitus sumiso es que orienta a las mujeres a que se atribuyan, en menor medida que los hombres, las competencias legitimas. Así Bourdieu expone un ejemplo clarificador al respecto, destacando que en las encuestas realizadas en la entrada de los museos, un buen número de mujeres expresa su deseo de ceder a su acompañante masculino la tarea de responder a las preguntas. Ante esta situación, Bourdieu establece que «valdría la pena hacer un inventario de todas las conductas que demuestran la dificultad casi física que las mujeres tienen para entrar en las acciones públicas y librarse de la sumisión al hombre como protector, tomador de decisiones y juez" (Bourdieu, 1999, 9).

Con relación a esta sumisión resultan muy ilustrativas algunas de las conclusiones obtenidas por E. Goffman al analizar la representación de la feminidad en los anuncios publicitarios. De entre las conclusiones extraídas subrayamos que las fotografías publicitarias suelen mostrar siempre a un hombre que instruye a una mujer, y no al contrario. Otro ejemplo es que la mujer es retratada, con frecuencia, en posición subalterna o de asistida. El hombre, por el contrario, simboliza su posición superior por su estatura más alta, representando una postura siempre protectora. También destaca que en los anuncios publicitarios la mujer "parece a menudo despegada de lo que la rodea (tener "la cabeza en otro sitio"), a pesar de estar al lado de un hombre, como si la vigilancia de él, preparado para enfrentarse a todo lo que pueda ocurrir, bastase por los dos" (Goffman, 1991, 151). Estas escenas publicitarias, que se repiten por doquier, ejemplifican la relación poder-sumisión que se establece entre los géneros.

Otra propiedad que configura el habitus de género femenino, e íntimamente relacionada con el punto anterior, es la cualidad expresiva. Habitus expresivo, por tanto, que define a la mujer atendiendo a sus aptitudes de relación (buena madre, buena esposa). Mientras en los hombres se produce una necesidad de logro y de 
afirmación social ( habitus impersonal), en las mujeres se destaca el predominio del mundo subjetivo. Por contraposición al habitus impersonal, y siguiendo nuevamente a Gilligan, hemos de destacar un conjunto de disposiciones (habitus) expresivas que tienen su origen en el papel realizado por la mujer a lo largo de todo el ciclo vital. Papel que les ha hecho desempeñar el rol de alimentadoras, cuidadoras y tejedoras de un conjunto de relaciones humanas de las que ellas, a su vez, pasan a depender.

Pues bien, esta clisposición expresiva genera la obligación de atender, cuidar y una responsabilidad constante por aliviar los conflictos, evitando siempre que alguien salga dañado y en caso de que tenga que haber una víctima que sea ella misma. Así “cada mujer se siente responsable ante los demás y sobre todo se siente responsable de las demás personas con quienes comparte el mismo contexto" (Gil Calvo, 1997, 138). Desde este punto de vista, se produce una orientación hacia otras personas concretas y no hacia un otro generalizado y abstracto, que se correspondería con la orientación propia del género masculino. El punto de vista del "otro concreto" ve a cada ser humano como una persona dotada de una historia particular y concreta, con una identidad particular. En este planteamiento abuscamos comprender las necesidades del otro, sus motivaciones y lo que desea. Nuestra relación con el otro está gobernada por normas de equidad y de complementariedad recíproca" (Beriain, 2000, 160).

Nos encontramos, por tanto, con una perspectiva de atención personal, en palabras de C. Gilligan, que se distingue por unas prácticas contextualizadas, particulares y concretas. De manera que, por ejemplo, las mujeres ven a sus amigas y amigos como personas concretas, de carne y hueso, identificándolas en sus contextos específicos. Como afirma E. Porter (1999), las amistades de las chicas se caracterizan por las actividades comparticlas, por la comprensión mutua y por la aceptación de las diferencias en las opiniones y por el respeto de las mismas. Se establecen unas responsabilidades comprometidas y particulares que exigen una atención personal y especial. Como señalan Britton y Baxter (1999) los discursos que se centran en un "yo" individualizaclo y aislado representan mejor la experiencia de los hombres que de las mujeres, puesto que éstas basan su experiencia en la relación con otros y otras.

Conecta este habitus expresivo con la ética del cuidado descrita por Gilligan como la ideología moral propia del sexo femenino. Una ideología que se basa en el concepto de la no riolencia y en el reconocimiento de las diferencias de necesidad. Este habitus expresivo es el que concluce a la adopción de determinadas actitudes que forman parte de una identidad cle género fuertemente apegada al estereotipo que, descle la sociedad, se considera adecuado y deseable para las féminas. De ahí que las jórenes encuestadas para un estudio realizado por Ortega (1993), se encuentren dispuestas a esperar recompensas afectivas, lo que las lleva a considerar el ámbito doméstico como la esfera de su afirmación personal. La incorporación de la mujer al mundo laboral se está produciendo progresivamente, ahora bien, para las jóvenes se torna escasamente estimulante, por lo que el trabajo 
fuera del hogar, concluye Ortega, se ve como una actividad secundaria para la realización personal.

En este marco, el habitus de género femenino y masculino aparece como el elemento estructural generador de unas identidades de género que, porque están fuertemente adaptadas a lo que la sociedad establece como deseable y adecuado para cada uno de los géneros, hemos denominado como esquemáticas, usando para ello la terminología de la psicóloga Sandra Bem.

Llegados a este punto debemos establecer una pequeña pero importante matización en la conceptualización de la identidad de género. Así, oponemos lo que denominaremos como identidad de género esquemática a la identidad de género no esquemática. En el establecimiento de esta diferenciación interna del término nos hemos apoyado, aplicando el principio de transferencia analógica, en la teoría desarrollada por la psicóloga Sandra Bem. La novedosa aportación que esta prestigiosa investigadora proporcionó, a mediados de los años 70, fue el concepto de esquema de género. Dicho concepto hace referencia a las estructuras cognitivas que, presentes en todas las personas, se encargan de codificar y procesar la información proveniente de la realidad, aplicándole los patrones del género. A partir de este término S. Bem estableció una diferencia entre los sujetos muy esquematizados en función del género, cuyo rasgo primordial es que procesan la realidad y su propio autoconcepto según el esquema de género, de manera que aceptan e interiorizan los estándares típicos de la sociedad en la que viven. Por otro lado, destacan los sujetos escasamente esquematizados, que no recurren al esquema de género cuando procesan la información, de manera que, en función de la demanda situacional, son capaces de comprometerse en comportamientos efectivos a la situación contextual, sin tener en cuenta el ideal de feminidad/masculinidad que la sociedad ha establecido. A partir de esta diferencia nosotros aplicamos la oposición esquemático/no esquemático a cuestiones no puramente cognitivas referidas al procesamiento de la información, corriente psicológica en la que se apoya $S$. Bem, sino al conjunto de conductas, actitudes y formas de sensibilidad generadas por la identidad de género. En este sentido, destacamos la existencia de una identidad de género esquemática, fundada en aquello que, desde la sociedad, se considera apropiado para cada género. Una identidad fuertemente apoyada en los tradicionales ideales de feminidad/masculinidad. Por otra parte, se encuentra la identidad de género no esquemática, más equilibrada y que no se fundamenta en tales estereotipos. Identidad(es) flexible(s) que escasean y que, desde nuestro punto de vista, son el ideal a alcanzar.

A partir de ello, en las páginas que siguen centraremos nuestra atención en la identidad esquemática de género, analizando algunos mecanismos escolares que están influyendo en la definición de identidades esquemáticas. El habitus de género se conforma a través de procesos de socialización implícita llevados a cabo por diversas instituciones. Entre éstas destaca la acción de la familia y de la escuela. En las páginas que siguen subrayamos el papel que juega la institución escolar como espacio en el que se constituyen los esquemas cognitivos, emocionales! 
motivacionales que conforman el habitus de género, matriz estructurante de la identidad esquemática.

\section{Aportaciones para una Teoría de la Educación desde una Perspectiva de género}

\subsection{La escuela como lugar de intercambio e interacción social}

Sin lugar a dudas el campo escolar actúa como espacio o escenario en el que se ponen en juego una serie de elementos sociales y en el que la vida social se cristaliza. El sujeto en tanto que sujeto social se construye en las interacciones sociales que se producen en la vida cotidiana, de modo que en la edad infantil y adolescente la asistencia diaria a la escuela hace que ésta se constituya en espacio idóneo de encuentro e intercambio social. Y es que este hecho tiene una importancia determinante si tenemos en cuenta que en estas edades "sin la interacción social, ni puede adquirirse la competencia lingüística ni el resto de habilidades sociales" (García Carrasco y García del Dujo, 2001, 36). De modo que el tiempo escolar es fundamental para reconstruir la vida social y para servir de ensayo de las diversas conductas, comportamientos y actitudes que configuran la identidad de género esquemática.

Las creencias, aptitudes, valores, actitudes y comportamientos del profesorado y del alumnado influyen en la conformación de identidades esquemáticas, por cuanto son personas reales que asignan significados a lo que sucede en la escuela a partir de sus vivencias en esa institución. Niños y niñas "aprenden a partir de sus experiencias con las disposiciones sociales de la escuela, al mismo tiempo que aprenden de la enseñanza misma" (Dreeben, 1998, 109). Un aprendizaje que incluye información, creencias y formas de comportarse acordes a un género determinado. La escuela en cuanto ámbito de socialización secundario es una institución sumamente eficaz pues a través de ella se generaliza la interiorización de las disposiciones básicas que socialmente se asignan a cada género.

La educación y el sistema de enseñanza son instrumentos fundamentales de la continuidad histórica y, por tanto, tienden a producir y reproducir la integración tanto cognitiva como emocional al grupo de género al que se pertenece. La construcción de las masculinidades y las feminidades en las escuelas dista mucho del mero aprendizaje explícito de las normas sugeridas y que deben afrontar cada uno de los géneros. Al contrario, es un proceso de enorme complejidad que supone encuentros entre niños y niñas en plena evolución con una institución educativa poderosa que se encuentra ante un proceso de transformación y cambio.

En suma, la escuela es un marco adecuado para que el juego social se ponga en marcha y para que en ese juego social se formen los patrones estereotipados de lo que significa ser hombre y mujer en nuestra sociedad. Si tuviésemos que destacar un rasgo fundamental del proceso de socialización del género que se lleva a cabo en el seno de la institución escolar es que se trata de un proceso de inculcación implícito, una lenta familiarización con los comportamientos adecuados al género 
y que supone un dominio inconsciente del conjunto de conductas y formas de sensibilidad que forman parte del repertorio de un género determinado. Por tanto, en la interacción diaria en el campo escolar las identidades de género esquemáticas se adquieren de la forma más adecuada inconscientemente, interiorizándose sin que afloren a la conciencia más que de forma parcial. A su vez, esta interiorización solamente se puede realizar mediante un proceso de inculcación implícita que permite que esa adquisición no pase a través de la conciencia, de forma que no es memorizada sino establecida en un nivel prerreflexivo. La inculcación, que se realiza de forma implícita, provoca que los esquemas de percepción, apreciación y acción propios de un género determinado sean interiorizados de una manera casi natural.

\subsection{Mecanismos escolares responsables de la configuración de identidades esquemáticas de género}

Evidentemente, el primer aspecto que debemos destacar es que este proceso de inculcación implícito se realiza por la mediación de "otros significativos" para la persona que es objeto de ese proceso de inculcación, en nuestro caso profesores y profesoras desempeñan, junto a padres y madres, el papel de "otros significativos". En este sentido, afirmamos que los "otros significativos" seleccionan aspectos del mundo en función de la situación que ocupan en la estructura social, posición claramente determinada por su género, y también en virtud de sus idiosincrasias individuales, idiosincrasias que igualmente están influenciadas por el género de pertenencia. Además, al ser un proceso de inculcación en el que están comprometidas las personas que forman parte del mundo social del niño y de la niña, una característica a destacar de este proceso es su carácter afectivo y empático. Por ello, es algo más que un aprendizaje de carácter puramente cognitivo, pues se produce una conexión afectiva y expresiva con los "otros significativos". Esta identificación emocional es una característica importante para que ese proceso de inculcación implícita se realice eficazmente?

Otro aspecto que caracteriza la interacción social que se produce con "otros significativos" es que estos adultos están dotados de un gran poder para establecer modelos ideales de cómo los niños y niñas deben ser en el orden moral. De forma que "ser uno mismo es serlo en una trama de relaciones múltiples en donde actuamos como creemos ser, y somos como los demás esperan que seamos" (Ortega, Fagoaga y García de León, 1993, 36). En el caso concreto de la escuela, los niños y las niñas se muestran como los docentes esperan que ellos se comporten, pues son especialmente receptivos a aquellas prescripciones que responden a las expectativas

9. Sobre el particular se pronuncian nuevamente GarCía CARRASCo y GARCía Del Dujo (2001) al señalar que la fractura tradicional que se produce entre la razón y la emoción impide "ver los mecanismos mediante los que interactúan la afectividad y el entendimiento y con los que se construye la identidad personal y comunitaria" (García Carrasco y García del Dujo, 2001, 33). 
de las personas que forman parte de su mundo inmediato. Así, por ejemplo, los niños tienden a desarrollar habilidades físicas y a ser autónomos si nosotros los adultos esperamos de ellos esos resultados. En los niños y niñas el sentido de valor de sus acciones está profundamente influido por la valoración que de las mismas realizan los adultos que les rodean. Además, una desventaja injusta inherente a la situación del niño y la niña, pues aunque en el proceso de socialización no son simples espectadores pasivos, son los adultos quienes disponen las reglas del juego (Berger y Luckman, 1986).

De este modo las disposiciones básicas (habitus) de las respectivas identidades de género esquemáticas se comienzan a apreciar como formas seguras. Si se alcanzan correctamente, son reconocidas por los adultos, en este caso docentes, como modos de ser adecuados, por lo que los niños y las niñas que los adquieren creen apropiarse, a los ojos de las personas que los rodean, de un estatus elevado. Ello, evidentemente, les llena de satisfacción. Los niños y las niñas ven una conducta apropiada a su género como moralmente requerida, se ajustan a ella e incluso expresan sentimientos de censura hacia los niños y niñas que se desvían de ella. A todo ello se ha de unir la importancia del autoconcepto pues si uno se comporta de conformidad a lo esperado por la sociedad, siguiendo las directrices del esquema de género, los adultos le reforzarán positivamente sus componentes tipificados, por lo que su autoestima y autoconcepto serán mayores.

Con relación a estas cuestiones queremos destacar el caso expuesto por Davies referente a una niña de preescolar de cuatro años. Esta niña era ya un caso excepcional pues reconocía su deseo de comportase de acuerdo con las formas de ser masculinas, cuestión totalmente inaudita porque los niños y niñas de esta edad se adaptan a lo que la sociedad establece como deseable y adecuado para los géneros; sin embargo, también aceptaba un deseo igual, o incluso mayor, de ser socialmente competente. Debía, por tanto, esforzarse por mantener su competencia social, pues comportarse incorrectamente con respecto al género era experimentado como una interrupción en el logro de la competencia social mencionada. Se producía, en palabras de Davies, una falta de práctica en los comportamientos del género opuesto, y esto se combinaba con la idea de que siempre que ensayaba algún comportamiento "incorrecto" parecía extravagante y poco convincente. La niña no era capaz de afirmar que prefería ciertos comportamientos "masculinos" en y por sí mismos, pues ello suponía poner en duda su comprensión del funcionamiento de las categorías de género y de su propio éxito en tanto que persona dotada de un género. Esta situación era resuelta por la niña mostrando una preferencia por relacionarse con las personas que legítimamente podían tener acceso a aquellas actividades que ella misma deseaba poner en práctica, es decir, los niños. Observamos, por tanto que se produce un sentimiento de estupidez que hace vergonzoso comportarse como el género opuesto. Pues bien, este sentimiento «llega a formar parte de la estructura psíquica, tanto que puedes llegar a reírte de ti mismo cuando te observas comportándote como el sexo opuesto" (Davies, 1994, 100). Nuevamente observamos la misma reacción cuando Davies narra a los niños y 
niñas de una clase de educación infantil cuentos de temática feminista, es decir, relatos en los que los papeles tradicionales que se asignan a los personajes, y que están fuertemente estereotipados por razón de género, son alterados con el objetivo de proponer modelos femeninos y masculinos que rompen con dicho estereotipo. La reacción unánime de los niños y niñas era la incredulidad, incluso el rechazo hacia un tipo de relatos que no se adaptaban a las convenciones sociales establecidas, de modo que, por ejemplo, la idea de que una princesa podía rescatar al príncipe de las garras del dragón malvado suponía una ruptura del esquema mental poseído que originaba los sentimientos anteriormente descritos. Y todo ello en un mundo que se define "de fantasía", de manera que les resultaba más fácil aceptar la idea de que existía un mundo de príncipes, princesas y dragones que el hecho de que la pertenencia a uno u otro género no lleva implícita la obligación de comportarse de una manera determinada.

Por otra parte, queremos aclarar que la experiencia de inculcación en la cual la niña y el niño se hallan inmersos no es una experiencia personal, sino interaccional pues solamente por la mediación de los otros, es decir, a través de un proceso de interacción constante con las personas que nos rodean, la identidad de género esquemática se forma en tanto que disposición básica del individuo. No es una experiencia personal, aunque sí subjetiva pues supone un posicionamiento del propio agente con relación a un conjunto de circunstancias que son percibidas y aprehendidas por el individuo como referido a él. El proceso de inculcación de las identidades de género esquemáticas se realiza mediante la interacción constante con el entorno escolar, interacción que provoca que la persona construya, de forma activa, los significados que organizan sus percepciones y experiencias. Niños y niñas abordan una valoración espontánea de sí mismos y de los demás, además, tienen una tendencia cognitiva a atribuirse un valor a sí mismos, a comparar el suyo con los demás y a medir el de otras personas. En esa valoración que realizan de sí mismos influye tanto el juicio realizado sobre la propia figura corporal como la autoclasificación como hombre o mujer basada en aquellos aspectos que han ido conformando culturalmente a mujeres y hombres. En suma, apreciamos que se trata de una experiencia, a la vez, interaccional y subjetiva, en la que niños y niñas interiorizan las posibilidades y los límites que conlleva cada género y refuerzan y mantienen la construcción social de los géneros.

De gran importancia en este contexto de interacción con "otros significativos" es la adjetivación que los docentes realizan de chicos y chicas, basándose para ello en pares de adjetivos antagónicos como, por ejemplo, pasivo/activo, tranquilo/agresivo, para configurar la personalidad básica y diferencial de ambos géneros. A partir de aquí se reproduce la adjudicación de un determinado rasgo de personalidad a un alumno o alumna, proceso que se ajusta más a una necesidad de constatar un hecho asumido por la sociedad que a una comprobación de hipótesis formuladas a partir de la relación docente/estudiante. De esta forma, los enseñantes reproducen en las observaciones sobre chicos y chicas lo que la sociedad atribuye a los géneros. Las diferentes disposiciones de género son reforzadas en el 
campo escolar por parte de un profesorado compuesto por hombres y mujeres pertenecientes a una sociedad determinada y que han sido objeto de una socialización de género que les ha inculcado unas identidades de género esquemáticas. De modo que en su trabajo como docentes socializan, a través de mecanismos inconscientes, a niños y niñas en pautas de comportamiento diferencial en función del género. Un ejemplo que demuestra esta asignación diferencial realizada por los docentes y la socialización difusa que se lleva a cabo es la mayor permisividad que se adopta con relación a las conductas agresivas manifestadas por el género masculino. Y es que en la escuela "opera un código cultural que establece que todos los chicos pueden verse implicados en situaciones violentas por el mero hecho de serlo. Este código... separa los espacios masculinos de los femeninos, estableciendo unas fronteras que son muy difíciles de atravesar" (Rambla, Rovira y Tomé, 1999, 48). Este código cultural es reforzado, la mayoría de las veces inconscientemente, por unos docentes que han asignado a ambos géneros a polos distintos en la dicotomía tranquilidad (pasividad)/agresividad (actividad). Profesores y profesoras atribuyen un comportamiento diferencial a los géneros con relación a la agresividad, lo que lleva a aplicar, de forma distinta, normas de tolerancia, las cuales se categorizan de manera muy diferente en función del género del transgresor. A los niños se les permite hacer más ruido, chillar y pelearse; en cambio, está muy mal visto que sea una niña la que desarrolle este tipo de conductas agresivas. Nos encontramos ante una labor de socialización que busca acentuar en cada uno de los géneros aquello que define socialmente la identidad propia de cada uno, fomentando las prácticas que convienen y prohibiendo o desalentando los comportamientos inapropiados.

Esta distinta caracterización también es subrayada por X. Bonal para quien «los niños son considerados como activos, creativos, movidos, inmaduros, arriesgados, etc., mientras que las niñas son dóciles, formales, pasivas, complacientes, cuidadosas, etc." (Bonal, 1993, 142). Además, a las niñas se las potencia verbalmente para que se acicalen ${ }^{10}$ y para que se comporten de una manera delicada; mientras que a los niños se les tolera que griten y demuestren mayores dosis de agresividad, así como que sean más duros en su temperamento ${ }^{11}$.

Sin duda alguna los discursos del profesorado sobre los rasgos que caracterizan a niños y niñas nos dice mucho del conjunto de esquemas inconscientes que les permiten dar sentido y significado al mundo que les rodea a partir de patrones de género. De forma que los docentes implicados en la investigación en curso que

10. Sobre el particular destaca un ejemplo descrito por G. Arenas en el que se demuestra la importancia de resaltar al aspecto físico en las ninas. Así un profesor comentaba el aspecto de la ropa de una niña diciendo que: "Hola Teresa. iQué sombrero tan lindo!, has conseguido superarte, y llegar a la cima" (ARenas Fernández, 1996, 135).

11. Destaca nuevamente un comentario descrito por Gloria Arenas en el que un profesor trae un caracol en una caja y cuando una niña intenta tocarlo dice: “Uf, no lo toques tú, es muy viscoso. Que uno de los niños lo coja y lo saque fuera al jardín" (ARENAS FERNÁndez, 1996, 136). 
estamos realizando, y que ha sido mencionada en la introducción ${ }^{12}$, manifiestan verbalmente actitudes explícitas acerca de los rasgos que configuran o deben configurar la personalidad de ambos sexos, lo que suponía adjetivar a las ninas como más presumidas, preocupadas por atender a las necesidades del otro (habitus expresivo), maduras, tranquilas, pasivas y con un pensamiento más reflexivo; al tiempo que los niños son denominados con los calificativos de nobles, brutos, despreocupados, irreflexivos y activos. Además, la exteriorización de estas actitudes diferenciales sobre los géneros adquiere, con frecuencia, una configuración más sutil, no tan explícita pero igualmente reflejo de su estructura profunda. Un ejemplo muy clarificador al respecto nos lo proporciona una maestra de educación infantil que en un grupo de discusión y a la pregunta del entrevistador de si niños y niñas manifiestan conductas distintas en la vida cotidiana del aula, contesta lo siguiente: "Las niñas son como la plastilina, se amoldan a todo. Los niños son más como esquinas y aristas y les cuesta más amoldarse... los niños tienen más aristas y picos que hay que pulir" ${ }^{13}$. Expresiones de este tipo demuestran toda una concepción acerca de las diferencias de género, del rol desempeñado por unos y otras en el espacio social y del modo en que niños y niñas son percibidos por los docentes.

Este comportamiento diferencial de los docentes hacia los niños y las niñas no es necesariamente consciente. Corresponde a un comportamiento espontáneo, efectuado por personas dotadas de un habitus de género que les lleva a establecer diferencias con relación a los niveles de competencia relativos a los dos géneros. Estas convicciones son transmitidas por los docentes e interpretadas por los estudiantes. Evidentemente, este tratamiento diferencial, así como las diferentes actitudes y expectativas del profesorado influyen en el refuerzo de los habitus masculinos y femeninos. En este ámbito, es muy frecuente que el profesorado reconozca la existencia de actitudes y conductas discriminatorias respecto al género, pero «suele tener dificultades para detectarlas en su acción docente y se siente, por

12. La investigación que comentamos ha tenido como objetivo fundamental descubrir si las representaciones estereotipadas de lo masculino y femenino, presentes en la sociedad, se encuentran interiorizadas por parte del profesorado de segundo ciclo de educación infantil. Investigación de corte cualitativo, en la que se ha usado como técnica de recogida de datos los grupos de discusión. Se han realizado 7 grupos de discusión en otros tantos colegios del Principado de Asturias, con una media de 5 profesoras participantes. Un aspecto que es necesario destacar es que el uso del grupo de discusión, en tanto que técnica cualitativa básica, trae consigo que las conclusiones obtenidas no se puedan generalizar; no habiendo, por tanto, posibilidad de lograr datos que sean significativos desde un punto de vista estadístico. No obstante, ello no es obstáculo para que los resultados clerivados de la investigación sean útiles y válidos. Además, se ha atendido al criterio de heterogeneidad por cuanto al organizar los grupos se ha pretendido que hubiera una representación de colegios de zona rural y urbana, al tiempo que centros de titularidad pública, privada y concertada.

13. Hemos de comentar que a lo largo de todo el grupo de discusión la profesora había mantenido una posición no esquemática y afirmaba constantemente que no percibía ninguna diferencia de comportamiento entre niños y niñas, al tiempo que observaba una reciprocidad en todas las conductas. Cuando la discusión estaba finalizando, y al preguntarle cómo eran los niños y las niñas, decla ró lo que se recoge en la cita. 
lo tanto, poco responsable de unos hechos que atribuye de modo impreciso a la sociedad y a la cultura" (Freixas y Luque, 1998, 56). Sin embargo, hay expectativas diferentes para chicos y chicas. Así, J. Carbonell (1996) afirma que a los chicos se les exige más creatividad, competencia y un mayor rendimiento; además, existen respecto a los chicos unas expectativas superiores en cuanto a las capacidades para cursar disciplinas científicas y estudios técnicos superiores. Sobre este último aspecto destacan las conclusiones de Warrington y Younger (2000) quienes demuestran en su estudio que las chicas y los chicos, todavía en la actualidad, asumen que las ciencias, en particular las ciencias físicas, son una materia masculina. Las chicas asumen que las ciencias son difíciles pero, como afirman los autores mencionados, ello no es consecuencia de una diferencia en la capacidad para hacer frente a estas materias, sino que lo que se produce es una diferencia de acercamiento a estas disciplinas que es reforzado por la propia actitud de los docentes. Desde esta perspectiva, el estudio expone ejemplos de cómo los docentes de ciencias se mostraban condescendientes con las chicas, al tiempo que revelaban una oculta admiración, que se tornaba en orgullo, por aquellos muchachos que sin grandes esfuerzos eran capaces de desenvolverse adecuadamente en este contexto. Complementando estas afirmaciones debemos subrayar la opinión de B. Francis (2000) para quien tradicionalmente las ciencias, incluyendo bajo este calificativo a disciplinas tales como la matemática, la física o las ingenierías, se han percibido como un dominio masculino, al tiempo que las humanidades se han construido como materias femeninas. Esa configuración ha sido consecuencia de la asociación de las ciencias a rasgos tales como la racionalidad y la objetividad, mientras que las humanidades se han coligado a la emoción y la subjetividad. Observamos la clasificación de las materias en dos polos opuestos y la asignación a los mismos de características típicamente femeninas o masculinas. Esta asignación diferencial guía, de forma inconsciente, el discurso del profesorado ayudando a configurar expectativas distintas para unos y otras.

Para finalizar con el estudio de la percepción diferencial que los docentes tienen de los niños y niñas debemos exponer otro ejemplo clarificador. Éste nos lo proporcionan las últimas investigaciones que se están realizando en los países anglosajones para explicar la feminización del éxito académico, proceso que también se está produciendo en nuestro pais ${ }^{14}$. Para explicar el mayor logro femenino los docentes afirman que las chicas son más organizadas, están dotadas de estilos comunicativos más sofisticados, se esfuerzan más en la realización de los trabajos y se adaptan de una forma más adecuada a las demandas de la escuela. Por el contrario, los chicos son percibidos, y por tanto adjetivados, como más desordenados,

14. Para un análisis del mayor rendimiento académico manifestado por el género femenino en nuestro país, véase la siguiente publicación: Gómez Bueno et al. (2001). Referencias que analizan lal situación en los países anglosajones son las siguientes: SalisbuY, ReEs y Gorard (1999); Yol Xicik, Warrington y Williams (1999); Warkington y Younger (2000); Whitelaw, Milosevic y DaNilis (2000): Gorard, ReEs y Salisbury (2001). 
desmotivados y distraídos que las chicas, menos preocupados por la imagen que proyectan ante el grupo clase y menos dispuestos a dar prioridad al trabajo escolar (Younger, Warrington y Williams, 1999). Naturalmente aunque estas percepciones docentes de las características diferenciales de ambos géneros pueden responder a un dato objetivo, es decir, son definitorias de la realidad; están ayudando a configurar distintas expectativas de lo que se espera de unos y otras, y ello influye en la perpetuación de dos ethos, el masculino y el femenino, distintos y distantes. Ethos directamente relacionado con dos identidades de género diferentes. Por un lado, se nos muestra una idea de una alumna que desarrolla una buena voluntad y docilidad escolar, lo que supone que se pliega por completo a las normas escolares y se adapta de una manera más adecuada al modelo disciplinario propuesto por la escuela; $y$, por otra parte, un alumno que presenta un mayor desapego a las normas escolares. En suma, percepciones distintas de lo que los chicos y chicas son, puede actuar como elemento reforzador de expectativas diferentes para unos y otras, ayudando a configurar identidades esquemáticas.

En conclusión, el profesorado, con frecuencia de forma inconsciente, proyecta en su práctica cotidiana una visión de la masculinidad y de la feminidad y a través de refuerzos y omisiones está proporcionando pautas para que niños y niñas conozcan las disposiciones básicas de sus géneros respectivos; disposiciones que se reflejarán en conductas y comportamientos de género, pues tratarán de imitar todo lo que oyen y hacen los adultos y adultas que les rodean. Se transmiten, de este modo, unos códigos sociales de comportamiento que comunican mensajes sobre cuál es el modo más adecuado en que cada género se ha de comportar.

Además, a través de las prácticas discursivas también se aprenden estrategias conversacionales, por cuanto la persona escoge algunas y rechaza otras que también tiene a su disposición. Niños y niñas aprenden a hablar no sólo escuchando, sino también hablando y, por ello, presentando un habla determinada (capital cultural incorporado) en los campos más diversos de la práctica. En el caso concreto que nos ocupa, presentan un habla propia y característica de un género determinado. Desde este punto de vista, y puesto que niñas y niños aprenden a mantener conversaciones en interacción con personas de su entorno, desarrollan habilidades discursivas diferentes y, por tanto, incorporan un capital cultural distinto. Estas habilidades comunicativas se aprenden a través de la experiencia discursiva, una experiencia que, en el caso de los niños y niñas, se adquiere a partir de su inclusión en grupos de iguales que rara vez tienen un carácter mixto. Las niñas con las niñas y los niños con los niños es el paradigma que define la configuración del grupo de iguales, lo que generará estilos comunicativos diferenciales en función del género ${ }^{15}$.

15. Para un análisis de estos estilos comunicativos diferenciales por razón de género acúdase a: Tannen (1996), Martín Rojo (1996) y Bengoechea (1995) entre otros. 
JOSÉ VICENTE PEÑA CALVO Y M. DEL CARMEN RODRÍGUEZ MENÉNDEZ IDENTIDADES ESQUEMÁTICAS DE GÉNERO EN LA ESCUELA:

A PROPÓSITO DEL PRIMER ANIVERSARIO DE LA MUERTE DE PIERRE BOURDIEU

En este proceso de interacción destacamos la importancia del lenguaje en cuanto vehículo comunicativo esencial. Así "las palabras crean las cosas, porque establecen el consenso sobre la existencia y el sentido de las cosas" (Brunet y Morell, 1998, 185). Por ello, a través del lenguaje diversos esquemas se internalizan; además, para hacerse miembros aceptables de la sociedad niños y niñas "deben aprender a pensar y actuar de acuerdo con las formas lingüísticas aceptadas y conocidas. No se trata simplemente de una cierta habilidad..., sino de la adquisición de los medios gracias a los cuales se constituyen a sí mismos como personas que se relacionan con otras en el mundo social" (Davies, 1994, 17). Para esta autora, el lenguaje actúa como un recurso, pero también como una constricción, pues aunque permite constituirse como miembro de una sociedad, también limita los modos de ser disponibles. Los niños y las niñas juegan con las palabras, situándose dentro de una estructura narrativa, a través de la cual se configura la aceptación de sí mismos en cuanto personas dotadas de un género masculino o femenino. El lenguaje opera como simbolizador práctico de las ideas sobre el género, pues en las prácticas discursivas que aprenden niños y niñas se hallan contenidos los términos dicotómicos de la feminidad y la masculinidad, así como el lenguaje metafórico que también ayuda a construir el género. Todo ello se va inculcando progresivamente en las mentes de niñas y niños.

Las ideas contenidas en el párrafo anterior nos permiten incluir un aspecto de suma importancia en el desarrollo del juego social que se produce en el campo escolar. Este aspecto está relacionado con las interacciones que se producen entre iguales. Sin lugar a dudas, en los procesos de interacción y de juego social que se originan entre niños y niñas un factor que tiene una importancia determinante en el desarrollo de dichos procesos son las propias actitudes de alumnos y alumnas. Niños y niñas llegan a la escuela después de haber interiorizado una serie de esquemas sociales relativos a los papeles de género, y estos esquemas van a guiar los procesos interactivos en los que se vean envueltos. De forma que, del mismo modo que profesores y profesoras adjetivan a sus estudiantes, niños y niñas tienen interiorizados unos patrones diferenciales que aparecen definidos en la dirección de los contenidos de los modelos de género. Además, puede ocurrir que esa interiorización de los papeles de género no haya tenido la oportunidad de ponerse en juego en el seno familiar, de manera que será la escuela el espacio social en el que aparezcan y adopten una configuración determinada. De ahí la importancia, como estableceremos en la conclusión a este artículo, del papel de los docentes para intervenir, orientando y reconduciendo el juego social que se desarrolla en el aula y en el patio de recreo para permitir el ensayo de conductas no estereotipadas por razón de género.

Como demuestra la investigación realizada por Bonal y Tomé (1996), en la que se preguntaba a niños y niñas que valorasen lo mejor y peor de ser chico y chica, las respuestas referidas a cada sexo ponen de relieve que niños y niñas acceden a la institución educativa con actitudes diferenciales acerca de lo que significa ser hombre o mujer. Así, los niños valoran de sí mismos atributos y cualidades relacionados 
con la fuerza física y, especialmente, con lo que, en la época en que vivimos, otorga el estatus de la masculinidad: jugar bien al fútbol. Lo negativo del hecho de ser hombre se localiza en la transgresión de las normas escolares o sociales. Ser agresivo o ser castigado suman el $70 \%$ de las respuestas, lo que revela también que son los niños quienes están en disposición de poder transgredir las normas. Por otra parte, en lo mejor de ser niña destacan dos dimensiones: la valoración del cuerpo y la buena disposición para el estudio. Además, un 15\% de las niñas responde que lo mejor de ser niña es poder tener hijos e hijas y ser ama de casa, con lo que parece significativa la interiorización del rol de "madre-esposa cuidadora" (babitus expresivo). También señalamos que la valoración positiva del cuerpo se acompaña de una visión negativa de su cuidado. Así, aunque el culto al cuerpo es una ventaja desde el punto de vista estético también supone un sacrificio y una dependencia para las niñas, al tiempo que es el elemento clave en torno al cual se configura la percepción de la mujer en cuanto "ser percibida", "cuerpo para el otro" destinada a ser mirada y admirada. Por otra parte, la cuestión que concentra un mayor número de respuestas negativas con relación al hecho de ser niña es la debilidad (31,09\%); es decir, la imposibilidad de defenderse tanto desde un punto de vista físico como psíquico. Como vemos el propio "alumnado es cómplice de la jerarquía de géneros de forma inconsciente» (Bonal y Tomé, 1996, 44), pues adopta actitudes y expectativas que se corresponden claramente con dicha jerarquía. Naturalmente estas actitudes y expectativas se verán continuamente reforzadas en el juego de intercambios e interacciones sociales que se producen en el seno de la institución educativa.

Los dualismos de género, como parte integrante de su identidad social, son construidos activamente por los niños y las niñas en el seno de las interacciones que se realizan en la institución escolar. Desde esta perspectiva destaca el estudio realizado por Short (1993) en el que entrevistó a niños y niñas sobre sus creencias en torno a los estereotipos de género, concluyendo que si bien éstos rechazaron los papeles sociales tradicionales asignados a los géneros, el comportamiento en clase revelaba el ejercicio de comportamientos muy estereotipados y, al tiempo, discriminatorios del género masculino para con el femenino. Es más, algunos estudios (Francis, 1997; Skelton, 1997) demuestran que la configuración de identidades esquemáticas, centradas en torno al ejercicio de un papel dominante por parte de los chicos (habitus dominante) en oposición a un papel más pasivo y sumiso de las niñas ( habitus sumiso), también se produce en el juego social del aula. Sobre el particular señalar el estudio emprendido por Francis (1997). Esta autora entrevistó a niños y niñas de 7 a 11 años de edad y a partir de las mismas categorizó diversas formas de sexismo ejercidas en la interacción de chicos y chicas en el interior del campo escolar. Concretamente determina que la construcción del género como fuente de discriminación (dominante/dominada) se ejerce, en primer lugar, a través del abuso verbal mediante insultos y en las bromas basadas en la inferioridad femenina. Un ejemplo de este modo de proceder nos lo muestra la 
opinión de un chico que comenta: “los hombres tienen músculos verdaderos y las chicas músculos de papel.

Otras formas de ejercer conductas dominantes, descritas por Francis y que son reflejo de los sistemas de esquemas de percepción dominantes, son aquellas que se pueden recoger bajo la categoría de aabuso físico". Además, con relación a esta forma de discriminación resulta interesante comentar la apreciación de la autora que subraya cómo algunas de las chicas entrevistadas eran fuertes desde un punto de vista físico, pero la mayoría de ellas no tenían confianza en su propia capacidad corporal, por lo que no eran conscientes de que podían enfrentarse a los chicos. De modo que la mayoría de las chicas estaban intimidadas por la ostentación de la fuerza física masculina. En este marco, la disposición (habitus) sumisa femenina se manifiesta en una relación con el propio cuerpo insegura, el cuerpo se experimenta como un objeto frágil e inmóvil y esta percepción se construye en el seno de interacciones sociales de las que el juego social desarrollado en la escuela es una de sus manifestaciones. Por el contrario, en la construcción de la masculinidad, y en particular de los esquemas del habitus dominante, tiene una importancia determinante, en cuanto mecanismo de refuerzo, la demostración de la capacidad corporal y de la fuerza física que se posee ${ }^{16}$.

Por último, y en íntima relación con las ideas que se acaban de manifestar, Francis establece que otra forma de ejercer la dominación, lo que supone la configuración de identidades esquemáticas, es a través de la exclusión de las chicas de determinadas actividades y juegos. El ejemplo más común es el referido al rechazo masculino a permitir que las chicas jueguen al fútbol, basándose este rechazo en la afirmación de que las chicas no saben jugar. Además, a través del estudio de Francis se pone de manifiesto que cuando las chicas fueron aceptadas para jugar lo hacían con el permiso explícito de los varones, al tiempo que esta posibilidad fue presentada como un favor que se les concedía. Sobre el particular, son muchas las investigaciones (Thorne, 1993; Bonal, 1995; Grugeon, 1995; Renold, 1997; Connell, 1998) que demuestran que los niños sienten el espacio de juego como propio, lo acaparan y juegan a perseguirse activamente. Utilizan la pelota y el fútbol como medio para proceder a ese acaparamiento, de manera que X. Bonal (1995) concluye que el juego dominante es el fútbol, que no deja espacio para otros juegos, genera actitudes violentas por parte de los niños y hace que el espacio sea utilizado, básicamente, por los varones. Como afirma R. W. Connell (1998), el deporte es uno de los vórtices de la masculinidad pues mezcla poder, actividad, simbolización y emoción. La cultura del patio de recreo está organizada, en nuestro país, en torno a un deporte muy concreto: el fútbol. Éste define directamente un modelo de actuación competitiva y dominante como la forma más adecuada de

16. Para quien desee realizar un análisis detallado del sexismo ejercido a través del abuso verbal y físico acúdase, además, a las siguientes referencias: WoOdS y HAMMERSLEY (1995), ASKEw y Ross (1991), García Colmenares (1994), López Puig (1992), Whitney y Smith (1993), LaRkin (1994), entre otras. 
IDENTIDADES ESQUEMÁTICAS DE GÉNERO EN LA ESCUELA:

A PROPÓSITO DEL PRIMER ANIVERSARIO DE LA MUERTE DE PIERRE BOURDIEU

masculinidad ( habitus viril y dominante) e, indirectamente, margina otros modos de jugar y utilizar el espacio de juego que se corresponden con el modelo de actividad propiamente femenino ${ }^{17}$. Sobre el particular destaca un estudio etnográfico, citado por Connell (1998), realizado en un instituto de Tejas, en EE.UU. En éste se proporciona una descripción del equipo de fútbol americano de dicho instituto. Se señala que la actividad desarrollada en torno a dicho equipo supone un refuerzo constante de la identidad propia de cada género. De manera que jugadores y animadoras se convierten en modelos de deseabilidad social para chicos y chicas respectivamente. Modelos que refuerzan la configuración de identidades esquemáticas de género.

En suma, en estas páginas hemos descrito algunos mecanismos escolares que influyen en la configuración de los esquemas del habitus que configuran el soporte de las identidades esquemáticas de género. En particular, hemos centrado nuestra atención en la dinámica del juego social que se lleva a cabo en el interior de la institución escolar, pues a través de ella la configuración de los roles sociales del género se refuerza. El habitus primario adquirido previamente en el hogar es continuamente reforzado a través de una institución educativa que debe tomar conciencia del papel que desempeña en la reproducción de estereotipos como primer paso para acabar con las diferencias de género.

\section{Conclusiones}

La acción social necesaria para poder socavar el actual orden social de género exige la implicación de los agentes individuales y de las instituciones sociales. Evidentemente, es en este nivel donde la institución educativa puede demostrar su valía. Pero, ¿qué puede hacer la escuela? Como señala M. J. Izquierdo "de entre los compromisos y responsabilidades que podemos asumir, hay uno que quisiera destacar: la modelación de la subjetividad de nuestros alumnos. No quiero con ello decir que seamos responsables únicos, sino subrayar que también nosotros lo somos" (Izquierdo, 1997, 10). En este sentido, ya hemos señalado la importancia de la institución educativa en la construcción de la identidad esquemática de género. Ahora bien, también hemos de destacar que niños y niñas han sido objeto de una socialización familiar, que ha sido el germen de dicha identidad. Por tanto, la subjetividad de los niños y las niñas nos llega ya construida en las relaciones familiares. Éste es un fenómeno con el que se tiene que contar, pero si admitimos que las personas estamos en un proceso permanente de construcción, siempre hay una

17. Dado que excede de los límites marcados mostramos a continuación algunas referencias que pueden revisarse para analizar el modo en que se construye una masculinidad esquemática a través del deporte en general y del fútbol en particular: SKelTon (2000), Swain (2000), Martino (1999). La lectura de estos artículos permite tener una primera toma de contacto con este tema que se puede complementar con la abundante bibliografía que aportan. 
JOSÉ VICENTE PEÑA CALVO Y M. DEL CARMEN RODRÍGUEZ MENÉNDEZ IDENTIDADES ESQUEMÁTICAS DE GÉNERO EN LA ESCUELA:

A PROPÓSITO DEL PRIMER ANIVERSARIO DE LA MUERTE DE PIERRE BOURDIEU

aportación que hacer, aunque sea limitada. La escuela contribuye a construir la subjetividad y el peso de la familia no nos releva de la responsabilidad que tenemos como docentes. Los géneros han de cambiar, social y psíquicamente, y la escuela, en cuanto ámbito de socialización, puede ayudar en la construcción de identidades de género no esquemáticas.

Ahora bien, hay que tener en cuenta que la intervención socioeducativa que se puede realizar desde la escuela está limitada por la influencia ejercida por la sociedad en general, y por la institución familiar en particular. De ahí que sea necesario una remodelación profunda que vaya más allá de lo que se construye a partir de la acción educativa. Y es que la realidad demuestra que la implementación de un cambio en la construcción del género conlleva un largo y difícil proceso de transformación de las personas y de las instituciones, incluida la escuela.

En este sentido, lo que podamos lograr en el ámbito escolar dependerá de muchos otros aspectos; como, por ejemplo, en qué medida la concepción que propugnamos es aceptada por las autoridades educativas, por los docentes, por los padres y madres y por la opinión pública en general. De manera que el cambio no ha de afectar solamente a la institución educativa, sino que ha de ir acompañado de transformaciones en el terreno de la economía, familia, derecho, etc. Las transformaciones que se propongan no pueden ser realizadas por una única instancia, en este caso la escuela, pues también son necesarias profundas innovaciones en la familia y en la sociedad.

La escuela no puede resolver, por sí sola, las desigualdades que la propia sociedad genera y alimenta, pero tiene su propio papel y su parte de responsabilidad en la lucha por la construcción de identidades de género no esquemáticas. La educación escolar no puede hacer desaparecer los esquematismos de género, pero es una pieza esencial para reducirlos. En suma, la enseñanza es una institución necesaria, aunque no suficiente, para la conquista de un objetivo que afecta a creencias y comportamientos muy enraizados en las sociedades y en los distintos grupos humanos.

A partir de aquí planteamos una conclusión de carácter general para este artículo y ésta es que la escuela ha de constituirse en una institución realmente coeducativa. El sistema cultural dominante en nuestro marco educativo es el sistema cultural de la escuela mixta (Bonal, 1995), basado en el principio democrático de igualdad entre las personas y en la defensa de la educación conjunta de chicos y chicas como un compromiso básico de la institución escolar. Desde esta perspectiva, el profesorado no concibe la masculinidad y la feminidad como construcciones sociales, sino como consecuencias naturales que se corresponden con el sexo de los agentes. Como máximo, el profesorado interpreta la masculinidad y la feminidad como una consecuencia del proceso de socialización familiar, proceso sobre el que la escuela no tiene ninguna responsabilidad. En el discurso docente no se piensa que la escuela contribuye a la definición de los géneros. En consecuencia, el género no es una variable relevante para la orientación de la práctica profesional.

(c) Ediciones Universidad de Salamanca

Teor. educ. 14,2002 , pp. 235-263 
JOSÉ VICENTE PEÑA CALVO Y M. a DEL CARMEN RODRÍGUEZ MENÉNDEZ

IDENTIDADES ESQUEMÁTICAS DE GÉNERO EN LA ESCUELA:

A PROPÓSITO DEL PRIMER ANIVERSARIO DE LA MUERTE DE PIERRE BOURDIEU

En contraposición a esta concepción de la escuela mixta oponemos, y siguiendo a X. Bonal (1995), un sistema cultural de colegio coeducativo. Tipo ideal al que se ha de tender y en el cual la variable género es relevante para la organización y orientación de la acción educativa. Desde esta perspectiva, la escuela mixta es vista como una institución que contribuye a la producción y reproducción de las disposiciones básicas (habitus) de la identidad esquemática de género. Por ello, el objetivo fundamental de la escuela coeducativa ha de ser "educar a las alumnas y a los alumnos, desde un concepto nuevo de persona, partiendo de su individualidad y al margen de los estereotipos sexistas" (Urruzola, 1995, 64).

En este marco, algunos autores (Shaw, 1995; Brutsaert, 1999) han demostrado que las adolescentes se perciben a sí mismas como personas más consistentes con los papeles sociales asignados a la feminidad en la escuelas integradas, aquellas en las que hay una presencia de chicos y chicas, que en las segregadas, compuestas solamente por chicos o por chicas. La razón que se argumenta para explicar esta situación es que en las escuelas integradas las chicas usan el rol femenino tradicional en tanto que mecanismo de defensa que les permite reducir la ansiedad ante la presencia de los chicos, es decir, sienten la necesidad de adecuarse al estereotipo como medio de reducir el estrés y la tensión escolares. Si a ello unimos otros estudios (Turner, 1999; Brutsaert y Van Houtte, 2002) que demuestran que el sentido de pertenencia a un grupo clase se desarrolla más en las chicas de escuela segregadas y de que éstas también favorecen el desarrollo de las habilidades interpersonales y permiten un mayor ajuste psicológico. Todo ello puede incitar a establecer la conclusión de que es necesario volver a separar a los chicos y las chicas en instituciones escolares distintas.

Sin embargo, desde nuestro punto de vista, el desarrollo de una personalidad más equilibrada en términos de valores masculinos y femeninos se debe perseguir en el interior de instituciones integradas, pues solamente en el seno de las mismas chicos y chicas pueden interactuar y ensayar conjuntamente nuevas formas de abordar su identidad de género. Esta actuación docente se debe emprender ya a edades tempranas, coincidiendo con los años de la educación infantil, momento clave en el inicio de las preferencias tipificadas por género en cuanto a amistades y juguetes (Jacklin y Lacey, 1997).

En suma, los comportamientos transgresores necesitan ser considerados como comportamientos normales, y de ahí la importancia del profesorado ofreciendo modos y modelos de ser que rompan con los esquemas de género y favoreciendo el ensayo de conductas que vayan más allá de los roles establecidos para ambos géneros. A ello se ha de unir la necesidad de que en la clase se examinen, desde una perspectiva crítica, las construcciones de la masculinidad y la feminidad, demostrando a los niños y las niñas la posibilidad de trascender estas visiones del género. 


\section{BiBLIOGRAFÍA}

Álvarez SOuSA, A. (1996) El constructivismo estructuralista: la teoría de las clases sociales de Pierre Bourdieu, Revista Española de Investigaciones Sociológicas, 75, 145-172.

Álvarez-Uría, F. y Varela, J. (eds.) (1986) Materiales de sociología crítica. Madrid, Ediciones de la Piqueta.

ARENAS Fernández, M. G. (1996) Triunfantes perdedoras. Investigación sobre la vida de las niñas en la escuela. Málaga, Universidad de Málaga, Instituto Andaluz de la Mujer.

Askew, C. y Ross, C. (1991) Los chicos no lloran. El sexismo en educación. Barcelona, Paidós (trabajo original publicado en 1988).

Bem, S. L. (1981) Gender schema theory: a cognitive account of sex typing, Psychological Review, 88, 354-364.

- (1983) Gender schema theory and its implications for child development: raising genderaschematic children in a gender schematic society, Signs, 8, 598-616.

Bengoechea, M. (1995) Mujeres/hombres: el conflicto entre dos culturas, Revista de Occidente, $170-171,120-136$.

BERGER, P. y LUCKMAN, Th. (1986) La construcción social de la realidad. Madrid, Amorrortu.

Beriain, J. (2000) El ser oculto de la cultura femenina en la obra de Georg Simmel, Revista Española de Investigaciones Sociológicas (REIS), 89, 141-180.

Bonal, X. (1993) La discriminación sexista en la escuela primaria, Signos. Teoría y Práctica de la educación, 8/9, 140-147.

- (1995) El professorat com a agent de canvi (microforma): una experiencia de recerca-acció en coeducación. Barcelona, Universitat Autónoma de Barcelona.

Bonal, X. y Tomé, A. (1996) Las representaciones de lo masculino y de lo femenino en el alumnado de educación primaria. Análisis y efectos sobre el profesorado, Signos. Teoría y Práctica de la Educación, 16, 42-48.

Bourdieu, P. (1991) El sentido práctico. Madrid, Taurus (trabajo original publicado en 1980).

- (1996) Cosas dichas. Barcelona, Gedisa (trabajo original publicado en 1987).

- (1999) La dominación masculina. Extraído de la página Web: http://www.udg/mx/laventana/libr3/bourdieu.html.

- (2000) La dominación masculina. Barcelona, Anagrama.

Bourdieu, P. y Waceuant, L. J. D. (1994) Per a una sociología reflexiva. Barcelona, Herder (trabajo original publicado en 1992).

BRITTON, C. y BAXTER, A. (1999) Becoming a mature student: gendered narratives of the self, Gender and Education, 11 (2), 179-193.

Brunet ICART, I. y Morell Blanch, A. (1998) Clases, educación y trabajo. Madrid, Trotta.

BRuTSAERT, H. (1999) Coeducation and gender identity formation: a comparative analysis of secondary schools in Belgium, British Journal of Sociology of Education, 20 (3), 343-353.

Brutsaert, H. y VAN HoutTe, M. (2002) Girl's and boy's sense of belonging in single-sex versus co-educational schools, Research in Education, 68-48.

CARBOnell, J. (1996) La escuela: entre la utopía y la realidad. Diez temas de sociología de la educación. Barcelona, Eumo-Octaedro.

CONNELL, R. W. (1998) Enseñar a los chicos: nuevas investigaciones sobre la masculinidad y estrategias de género para la escuela, Kikiriki, 47, 51-68.

Davies, B. (1994) Sapos y culebras y cuentos feministas. Los niños de preescolar y el género. Madrid, Cátedra. 
JOSÉ VICENTE PEÑA CALVO Y M." DEL CARMEN RODRÍGUEZ MENÉNDEZ

IDENTIDADES ESQUEMÁTICAS DE GÉNERO EN LA ESCUELA:

A PROPÓSITO DEL PRIMER ANIVERSARIO DE LA MUERTE DE PIERRE BOURDIEU

De Beauvoir, S. (1982) El segundo sexo. Buenos Aires, Siglo XX (trabajo original publicado en 1949).

DREEBEN, R. (1998) El currículum no escrito y su relación con los valores, Revista de Estudios del Currículum, 1 (1), 91-111.

Echebarría Echabe, A. y ValenCia Gárate, J. F. (1993) Identidad de género, ética protestante y atribución de causalidad, Revista de Psicología Social, 8 (2), 235-247.

EVENS, T. M. S. (1999) Bourdieu and the logica of practice: is all living Indian-giving or is "generalized materialism" not enough? For the memory of Louis Dumont, Sociological Theory, 17 (1), 3-31.

Francis, B. (1997) Discussing discrimination: children's construction of sexism between pupils in primary school, British Journal of Sociology of Education, 18 (4), 519-532.

- (1998) Oppositional positions: children's construction of gender in talk and role plays based on adult occupation, Educational Research, 40 (1), 31-43.

- (2000) The gendered subject: student's subject preferences and discussions of gender and subject ability, Oxford Review of Education, 26 (1), 35-48.

Freixas, A. y Luque, A. (1998) ¿A favor de las niñas? Notas en el debate sobre la escuela coeducativa, Cultura y Educación, 9, 51-62.

García Cancuini, N. (1998) De cómo Clifford Geertz y Pierre Bourdieu llegaron al exilio, Causas y Azares, 5 (7), 26-40.

García Carrasco, J. y García del Dujo, Á. (2001) La teoría de la educación en la encrucijada, Teoría de la Educación, 13, 15-43.

Garcí Colmenares, C. (1994) Socialización, estereotipos y agresividad, en VV.AA. Em busca de uma pedagogía da igualdade. Hacia una pedagogía de la igualdad. Actas da Universidade de Verão, Lisboa, 17-23 de julho de 1994. Lisboa, Comissão para a igualdades e para os direitos das mulheres, 237-247.

García Inda, A. (1995) La regla en la teoría de la práctica de Pierre Bourdieu, Revista Acciones e Investigaciones Sociales, 3, 243-267.

Gil Calvo, E. (1997) El nuevo sexo débil. Los dilemas del varón posmoderno. Madrid, Temas de Hoy.

Gilugan, C. (1985) La moral y la teoría. Psicología del desarrollo femenino. México, Fondo de Cultura Económica (trabajo original publicado en 1982).

GofFMAn, E. (1991) Los momentos y sus hombres. Textos seleccionados y presentados por Yves Winkin. Barcelona, Paidós.

Gómez Bueno, C.; Casares Fernández, M.; Cifuentes Martínez, C.; Carmona Bretones, A. y Fernández Palomares, F. (2001) Identidades de género y feminización del éxito académico. Madrid, Ministerio de Educación, Cultura y Deporte.

Gorard, S.; ReEs, G. y SalisBurY, J. (2001) Investigating the patterns of differential attainment of boys and girls at school, British Educational Research Journal, 27 (2), 125-139.

Grugron, E. (1995) Implicaciones del género en la cultura del patio de recreo, en WoODs, P. y Hammersley, M. Género, cultura y etnia en la escuela. Informes etnográficos. BarcelonaMadrid, Paidós-MEC, 23-48.

IzQuiERDO, M. J. (1997) Construcción de la subjetividad: estructura social y escuela, en IzQuierdo, M. J.; Sellarés, R.; Flecha, R. y Leal, J. Pedagogía crítica: malestar i ocultació. Lleida, Universitat de Lleida, 7-55.

JACKLIN, A. y LACEY, C. (1997) Gender integration in the infant classroom: a case study, British Educational Research Journal, 23 (5), 623-639.

Jenkins, R. (1993) Pierre Bourdieu. Londres, Routledge.

(c) Ediciones Universidad de Salamanca

Teor. educ. 14, 2002, pp. 235-263 
LARKIN, J. (1994) Walking through walls: the sexual harassment of highschool girls, Gender and Education, 6, 263-280.

López Puig, A. (1992) La agresividad en la infancia. Estudio empírico comparativo entre sexos, en Moreno Marimón, M. Del silencio a la palabra. Coeducación y reforma educativa. Madrid, Ministerio de Asuntos Sociales, 35-46.

MarTín Rojo, L. (1996) Lenguaje y género. Descripción y explicación de la diferencia, Signos. Teoría y práctica de la educación, 16, 6-17.

Martínez Sanmartí, R. y Pérez Sola, J. D. (1997) El gust juvenil en joc: distribució social del gust especificament juvenil de secundaria de Terrassa. Barcelona, Diputació de Barcelona.

MARTINO, W. (1999) "Cool boy's", "party animals", "squids and poofters": interrogating the dynamics and politics of adolescent masculinities in school, British Journal of Sociology of Education, 20, 239-263.

Mor, T. (1991). Appropiating Bourdieu feminist theory and Pierre's Bourdieu sociology of culture, New Literary History: a journal of theory and interpretation, 22 (4), 1017-1049.

MORGAN, D. (1999) Aprender a ser hombre: problemas y contradicciones de la experiencia masculina, en Luke, C. (comp.). Feminismos y pedagogias en la vida cotidiana. Madrid, Morata, 106-116 (trabajo original publicado en 1996).

MotTA-Lopes, L. P. (2003) Storytelling as action: constructing masculinities in a school context, Pedagogy, Culture and Society, 11 (1), 31-47.

Ortega, F.; FAGOAGA, C.; GARCíA DE LEÓN, M. A. et al. (1993) La flotante identidad sexual. La construcción del género en la vida cotidiana de la juventud. Madrid, Instituto de Investigaciones Feministas de la Universidad Complutense de Madrid-Consejería de la Presidencia, Dirección General de la Mujer.

PORTER, E. (1999) Mujeres y amistades: pedagogías de la atención personal y las relaciones, en LuKe, C. (comp.). Feminismos y pedagogias en la vida cotidiana. Madrid, Morata, 6686 (trabajo original publicado en 1996).

Rambla, X.; Rovira, M. y Tomé, A. (1999) Ocho trajes a medida. Modelos y resultados en cada país, Cuadernos de Pedagogía, 284, 48-53.

RENOLD, E. (1997) "All they've got on their brains is football": Sport, masculinity and the gendered practices of playground relations, Sport, Education and Society, 2, 5-23.

Salisbury, J.; Rees, G. y Gorard, S. (1999) Accounting for the differential attainment of boys and girls at school, School Leadership and Management, 19 (4), 403-426.

SÁNCHeZ de HorCAjo, J. J. (1979) La cultura. Reproducción o cambio. El análisis sociológico de Pierre Bourdieu. Madrid, Centro de Investigaciones Sociológicas.

SHAW, J. (1995) Education, gender and anxiety. London, Taylor \& Francis.

SHORT, G. (1993) Sex-typed behaviour in the primary school: the significance of contrasting expectations, Educational Research, 35 (1), 77-87.

SketTon, C. (1997) Primary boys and hegemonic masculinities, British Journal of Sociology of Education, 18 (3), 349-369.

- (2000) "A pasion for football»: dominant masculinities and primary schooling, Sport, Education and Society, 5 (1).

SwaIn, J. (2000) "The money's good, the fame's good, the girls are good": The role of playground football in the construction of young boys' masculinity in a junior school, British Journal of Sociology of Education, 21 (1), 95-109.

Tannen, D. (1996) Género y discurso. Barcelona, Paidós.

Thorne, B. (1993) Gender play: girls and boys in school. Buckingham, Open University Press.

(c) Ediciones Universidad de Salamanca

Teor. educ. 14, 2002, pp. 235-263 
IDENTIDADES ESQUEMÁTICAS DE GÉNERO EN LA ESCUELA:

A PROPÓSITO DEL PRIMER ANIVERSARIO DE LA MUERTE DE PIERRE BOURDIEU

Turner, G. (1999) Peer support and young people's health, Journal of Adolescence, 22, $567-$ 572.

UrRuzola, M. J. (1995) Espacios autónomos para los niños y las niñas en la escuela. Cuadernos de Pedagogia, 240, 64-65.

VAN Haecht, A. (1999) La escuela va a examen. Preguntas a la sociología de la educación. Buenos Aires-Madrid, Biblos-Miño y Dávila.

WARRINGTON, M. y YOUnGER, M. (2000) The other side of the gender gap, Gender and Education, 12 (4), 493-508.

Warrington, M.; Younger, M. y Williams, J. (2000) Student attitudes, image and the gender gap, British Educational Research Journal, 26 (3), 393-407.

Whitelaw, S; Milosevic, L. y Daniels, S. (2000) Gender, behaviour and achievement: a preliminary study of pupil perceptions and attitudes, Gender and Education, 12 (1), 87-113.

WhitNey, I. y SMITH, P. (1993) A survey of the nature and extent of bullying in junior/middle and secondary schools, Educational Research, 35, 3-25.

WoOds, P. y HaMmERSLEY, M. (1995) Género, cultura y etnia en la escuela. Informes etnográficos. Barcelona-Madrid, Paidós-MEC.

Younger, M.; WarRington, M. y Williams, J. (1999) The gender gap and the classroom interactions: reality or rhetoric?, British Journal of Sociology of Education, 20 (3), 325-341. 